\title{
Atmospheres of Polygons and Knotted Polygons
}

\author{
E J Janse van Rensburg ${ }^{\dagger} \S$, and A Rechnitzer ${ }^{\ddagger}$ \\ $\dagger$ Department of Mathematics and Statistics, York University, 4700 Keele Street, \\ Toronto, Ontario, M3J 1P3, Canada (rensburg@yorku.ca) \\ $\ddagger$ Department of Mathematics, The University of British Columbia, Vancouver, \\ B.C. V6T 1 Z2 (andrewr@math.ubc.ca)
}

\begin{abstract}
In this paper we define two statistics $a_{+}(\omega)$ and $a_{-}(\omega)$, the positive and negative atmospheres of a lattice polygon $\omega$ of fixed length $n$. These statistics have the property that $\left\langle a_{+}(\omega)\right\rangle /\left\langle a_{-}(\omega)\right\rangle=p_{n+2} / p_{n}$, where $p_{n}$ is the number of polygons of length $n$, counted modulo translations. We use the pivot algorithm to sample polygons and to compute the corresponding average atmospheres. Using this data, we directly estimate the growth constants of polygons in two and three dimensions. We find that

$$
\mu= \begin{cases}2.63806 \pm 0.00012, & \text { in } 2 \text { dimensions } \\ 4.683980 \pm 0.000042, & \text { in } 3 \text { dimensions }\end{cases}
$$

We also compute atmospheres of polygons of fixed knot type $K$ sampled by the BFACF algorithm. We discuss the implications of our results and show that different knot types have atmospheres which behave dramatically differently at small values of $n$.
\end{abstract}

Keywords: Lattice Polygons, Growth Constant, Knotted Lattice Polygons

\section{Introduction}

Lattice polygons are models of ring polymers with excluded volume and remains a mathematically rich and unsolved model in statistical mechanics $[9,10,6]$. This model poses a basic combinatorial question, namely, how many distinct polygons of length $n$ are there in the hypercubic lattice [15]? This question is related to the combinatorics of self-avoiding walks. If $c_{n}$ is the number of self-avoiding walks from the origin, of length $n$, then it is known that the limit

$$
\mu=\lim _{n \rightarrow \infty} c_{n}^{1 / n}
$$

exists in $d$ dimensions and $\mu>1$ in dimensions $d>1$ [16]. The constant $\mu$ is the growth constant of the self-avoiding walk, while $\kappa=\log \mu$ is the connective constant [4]. Several basic properties of $c_{n}$ are known: for example, it is known that the limit

$$
\mu^{2}=\lim _{n \rightarrow \infty} c_{n+2} / c_{n}
$$

exists $[25,26]$, but the stronger result $\mu=\lim _{n \rightarrow \infty} c_{n+1} / c_{n}$ remains unproven in the square or cubic lattice [14] (but is known for non-bipartite lattices such as the triangular lattice [30]). It has been also established that $c_{n+1}>c_{n}$ [36] in general. There are overwhelming numerical and other evidence that

$$
c_{n}=A \mu^{n} n^{\gamma-1}(1+o(1))
$$

$\S$ To whom correspondence should be addressed (rensburg@yorku.ca) 
where $\gamma$ is refered to as the entropic exponent of the self-avoiding walk.

Similarly lattice polygons have been the subject of much attention over the last fifty years. If $p_{n}$ is the number of polygons of length $n$ in the hypercubic lattice (counted up to translational invariance), then the limit

$$
\mu=\lim _{n \rightarrow \infty} p_{n}^{1 / n}
$$

is known to exist [15], and the growth constant of polygons is equal in numerical value to the growth constant of self-avoiding walks defined in equation (2). It is widely accepted that the asymptotic growth of $p_{n}$ is of the form

$$
p_{n}=A n^{\alpha-2} \mu^{n}(1+o(1))
$$

where $\alpha$ is the polygon entropic exponent or the specific heat exponent. The function $p_{n}$ satisfies

$$
\lim _{n \rightarrow \infty} \frac{p_{n+2}}{p_{n}}=\mu^{2}
$$

a result due to Kesten [25, 26]; see reference [30] for a simpler proof.

The numerical value of $\mu$ has been estimated for the square lattice using a variety of different methods, including computer enumeration and series analysis of lattice polygons, or grand canonical Monte Carlo simulations (in such simulations polygons are sampled from a Boltzman distribution over the lenghts of the polygons). The best estimates for $\mu$ have been obtained from computer enumeration and series analysis of polygons (rather than self-avoiding walks).

Series analysis for polygons $[23,24]$ gives $\mu$ and $\alpha$ to very high precision:\|

$$
\begin{aligned}
& \mu=2.63815852927 \pm 0.00000000001 \\
& \alpha=0.5000005 \pm 0.0000010 .
\end{aligned}
$$

The exponent $\alpha$ has also been estimated from conformal field theory and Coulomb gas methods, which gives the exact value $\alpha=1 / 2$ in two dimensions [33, 34].

Determining $\mu$ from self-avoiding walk data is not so successful. The best estimate for $\mu$ and the entropic exponent $\gamma$ in equation (4) obtained from self-avoiding walk data are

$$
\begin{aligned}
& \mu=2.6381587 \pm 0.0000007, \\
& \gamma=1.34372 \pm 0.00010 .
\end{aligned}
$$

as determined in reference [13].

Less precise estimates for $\mu$ and $\alpha$ are available in three dimensions. Clisby, Liang and Slade [5] estimated that

$$
\begin{aligned}
& \mu=4.684044 \pm 0.000011, \\
& \alpha \approx 0.24
\end{aligned}
$$

by collecting series data on the self-avoiding walk using the lace expansion. The exponent $\alpha$ has also been determined by field theoretic means [12, 27], giving $\alpha=0.237 \pm 0.002$.

In reference [40] a new statistic called the atmosphere of a self-avoiding walk was introduced. This statistic was shown (numerically) to converge to the growth constant $\mu$ as the length of the walk increases, and it is possible to determine estimates of $\mu$

|| Error bars and confidence intervals are those claimed in the original references. In this paper, we state our own error bars as $67 \%$ statistical confidence intervals. 
and $\gamma$ using canonical Monte Carlo simulation (of fixed length self-avoiding walks) [29]. The basic idea underlying the atmosphere of a self-avoiding walk is to measure the number of ways that an edge may be added to a walk of length $n$ to create a walk of length $n+1$.

The atmosphere of an (oriented) self-avoiding walk is defined as the set of edges which may be appended onto its last vertex to extend the walk by one step while maintaining self-avoidance. Denote the size of the atmosphere of a walk $w$ by $a(w)$. Then the mean atmosphere of walks of length $n$ is given by

$$
\langle a(w)\rangle_{n}=c_{n+1} / c_{n} .
$$

We shall often abuse our notation by using the term "atmosphere" to refer to both the atmosphere of the walk, and to the cardinality of the set of atmospheric edges. Equation (4) suggests that $\langle a(w)\rangle_{n}$ can be interpreted as a "local estimate" of $\mu$. Assuming that $c_{n}$ has an asymptotic form given by equation (4), then

$$
\langle a\rangle_{n}=\mu\left(1+\frac{\gamma-1}{n}+o(1 / n)\right) .
$$

Consequently, by obtaining precise estimates of the mean atmosphere of self-avoiding walks at various fixed lengths, estimates of both $\mu$ and $\gamma$ were obtained:

$$
\begin{aligned}
& \mu=2.63816 \pm 0.00006, \\
& \gamma=1.345 \pm 0.002 .
\end{aligned}
$$

These results verify digits obtained by computer and series enumeration [13] above in equation (10).

Previously, Monte Carlo estimates for $\mu$ and $\gamma$ have been made using grand canonical Monte Carlo algorithms which samples self-avoiding walks from a distribution over their lengths. The most well-known such algorithm is the BerettiSokal algorithm [3]. This algorithm has produced estimates of the connective constant as follows [35]:

$$
\mu=2.638164 \pm 0.000014,
$$

with error bars a combined $95 \%$ statistical confidence interval and an estimated systematic error due to uncertainties in the model. More recently the [11, 39] PERM algorithm has been used to find precise estimates of $\mu$ and $\gamma$ in three dimensions and higher.

In Section 2 of this paper we define a new statistic for polygons which plays the same role as the atmosphere statistic in walks. We show that the mean atmospheres of a polygon can be used to estimate $\mu$ and $\alpha$ in equation (6). Data were collected for polygons on the square and cubic lattices in two and in three dimensions, and by analysing the data (see Section 3), we estimate that

$$
\begin{aligned}
& \mu=2.63806 \pm 0.00012, \\
& \alpha=0.532 \pm 0.027
\end{aligned}
$$

in two dimensions, and

$$
\begin{aligned}
& \mu=4.683980 \pm 0.000042, \\
& \alpha=0.248 \pm 0.016
\end{aligned}
$$

in three dimensions. The estimate in three dimensions compare well to estimates in the literature. For example, reference [17] reported an (unpublished) estimate 


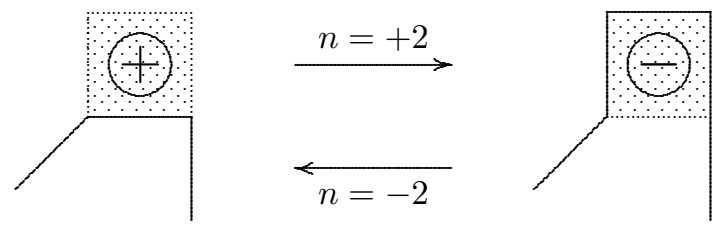

Figure 1. The atmosphere of a polygon: by adding two edges to the polygon around the square on the left, a polygon is found which has length increased by two. The collection of such squares incident with a polygon is its positive atmosphere. On the other hand, by instead deleting two edges to remove the square, we obtain a polygon which has length reduced by two. The collection of such squares incident with a polygon is its negative atmosphere.

$\mu=4.683907 \pm 0.000022$ due to A.J. Guttmann, while the best published estimate is due to self-avoiding walk enumeration using the lace expansion [5]. This gives $\mu=4.684043 \pm 0.000012$. The estimates in equation (19) above were obtained using relatively modest computer resources, and are consistent with those in reference [5].

In Section 4 we shift our attention to knotted polygons. The atmospheric statistic can be used to make numerical estimates of the ratios $p_{n+2}(K) / p_{n}(K)$ of the number of polygons of length $n+2$ and length $n$, and of knot type $K$. If the entropy of a polygon of length $n$ is defined by $\log p_{n}(K)$, then the logarithm of the ratio $p_{n+2}(K) / p_{n}(K)$ is the relative entropy of the polygon, and is a measure of the change in entropy with increasing $n$. For example, in Figure 8 we plot numerical estimates of the ratio $p_{n+2}(\emptyset) / p_{n}(\emptyset)$ for unknotted polygons. The data increase at small $n$ and quickly settles down to a constant. The increase at small $n$ implies that the rate of increase in $\log p_{n}(\emptyset)$ increases at small $n$. This is in constrast with polygons of fixed non-trivial knot type, as illustrated in for example in Figure 9 for trefoils. The rate of increase in $\log p_{n}\left(3_{1}\right)$ decreases with increasing $n$ at small values of $n$ before settling down to a constant within numerical variation. These data also indicate that the growth constant of a knotted polygon of fixed knot type is independent of knot type, but that the approach of $\left[p_{n}(K)\right]^{1 / n}$ to $\mu_{K}$ is from below for unknots, and from above for knotted polygons of fixed non-trivial knot type. We make some final comments in Section 5, and in Appendix I we show how the atmosphere statistic can be generalised to interacting models of polygons. This technique gives a method for directly estimating the limiting free energy of an interacting polygon.

\section{Atmospheres of polygons}

Let $\omega$ be a polygon, and let $e$ be an edge in the polygon. Incident with $e$ are $2(d-1)$ unit squares in the $d$-dimensional hypercubic lattice. These unit squares are all incident with $\omega$. In particular, any one such unit square $S$ has at least one edge $(e)$ in $\omega$. We say that $S$ is part of the positive atmosphere of $\omega$ if it has exactly one edge $(e)$ in $\omega$ and is otherwise disjoint with $\omega$. In this event, one may change $\omega$ so that it traverses the other three edges in the boundary of $S$ (see Figure 1). Since these edges are disjoint with $\omega$, a new self-avoiding polygon is obtained, which has length increased by two.

It is also possible that a unit square $S$ incident with $\omega$ has exactly three boundary edges in $\omega$. These three boundary edges form a three step walk in a $\sqcup$ - 


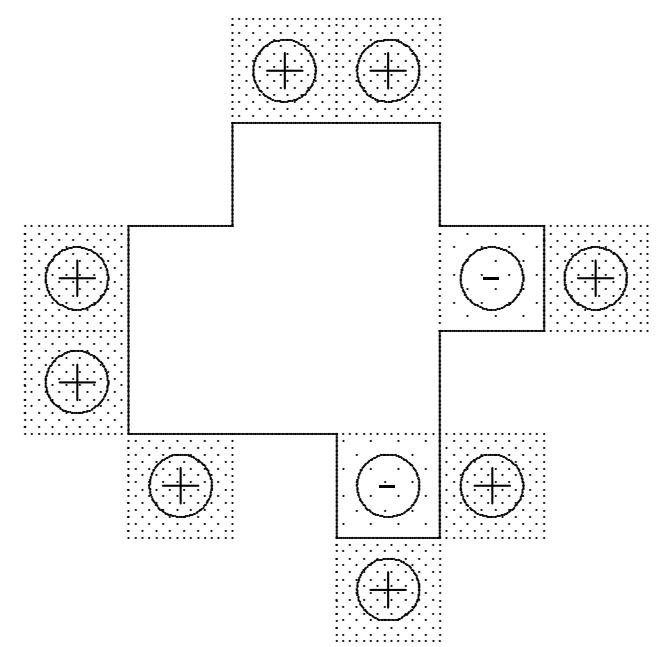

Figure 2. The atmosphere of a two dimensional polygon. The positive atmosphere is composed of the darker shaded unit squares. The negative atmosphere is composed of the lighter shaded unit squares. By adding two edges in the polygon so that it includes three edges of a positive atmosphere square, a new polygon is obtained which includes the atmospheric square as part of its negative atmosphere. By removing two edges from the polygon so that a negative atmopheric square is removed, a new polygon is obtained which includes the atmospheric square as part of its positive atmosphere.

conformation. If $e$ is the middle of the edges in this three step walk, then we say $S$ is a negative atmospheric square of $\omega$ incident with $e$. The collection of all such negative atmospheric squares incident with $\omega$ forms the negative atmosphere of $\omega$. The positive and negative atmospheres of a polygon are illustrated in Figure 2.

The action of adding edges to a polygon at a positive atmospheric square, or deleting edges at a negative atmospheric square, sets up a correspondence between polygons of length $n$ and of length $n+2$. Observe that the addition of two edges at a positive atmospheric square as in Figure 1 changes that positive atmospheric square into a negative atmospheric square. Similarly, if edges are deleted to cut a negative atmospheric square from a polygon as in Figure 1, then that negative atmospheric square is changed into a positive atmospheric square.

To examine this correspondence between negative and positive atmospheres, define $p_{n}\left(a_{+}, a_{-}\right)$to be the number of lattice polygons of length $n$ with $a_{+}$positive atmospheric squares, and $a_{-}$negative atmospheric squares. Observe that each polygon has at least two positive atmospheric squares located on the edges with lexicographic most and least midpoints. There are some polygons with zero negative atmospheric squares, for example, any polygon that is a geometric rectangle with sides lengths longer than two has no negative atmospheric squares.

The total number of ways that we can extend the length of a polygon by 2 edges in this way is $\sum_{a_{+} a_{-}} a_{+} p_{n}\left(a_{+}, a_{-}\right)$, and the result is in each case a polygon of length $n+2$. Each polygon of length $n+2$ obtained in this way has at least one negative atmospheric square, and is the image of exactly $a_{-}$polygons if it has $a_{-}$negative atmospheric squares. In other words, each polygon of length $n+2$ with $a_{-}$negative 


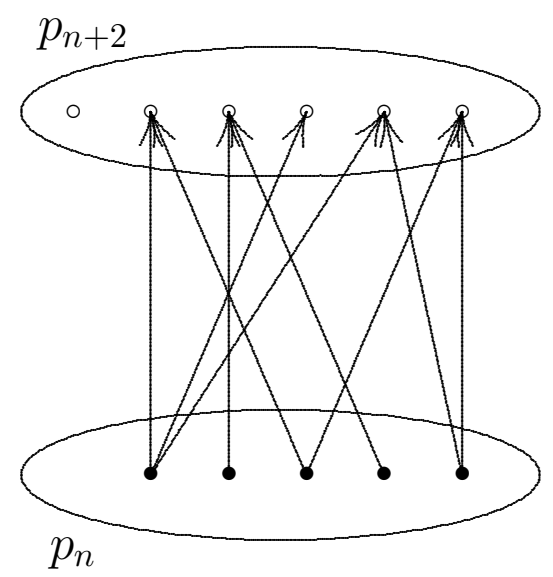

Figure 3. Polygons of length $n$ can be mapped to polygons of length $p_{n+2}$ by adding edges around an atmospheric square. In this schematic diagram, polygons of length $n$ are mapped to polygons of length $n+2$ along the arrows. Observe that each arrow corresponds to a positive atmospheric square on a polygon counted by $p_{n}$, or to a negative atmospheric square on a polygon counted by $p_{n+2}$. Each polygon of length $n$ may be mapped to a number of different polygons of length $n+2$ by adding the edges round a positive atmospheric square. The total number of arrows is equal to the number of ways edges can be added around positive atmospheric squares on polygons of length $n$. Conversely, by removing edges from a negative atmospheric square in a polygon of length $n+2$, a polygon of length $n+2$ is mapped to a polygon of length $n$. In the diagram above, moving against each arrow corresponds to the removal of edges from a negative atmospheric square. There may be some polygons of length $n+2$ without negative atmospheres. It follows that the number of arrows can be counted by either considering the addition of edges around positive atmospheric squares on polygons of length $n$, or by considering the removal of edges around negative atmospheric squares. This observation gives equation (23).

atmospheric squares are counted $a_{-}$times by the sum $\sum_{a_{+}, a_{-}} a_{+} p_{n}\left(a_{+}, a_{-}\right)$. Hence

$$
\sum_{a_{+}, a_{-}} a_{+} p_{n}\left(a_{+}, a_{-}\right)=\sum_{a_{+}, a_{-}} a_{-} p_{n+2}\left(a_{+}, a_{-}\right)
$$

where both sums are over all values of $a_{+} \geq 0$ and $a_{-} \geq 0$ since $\sum_{a_{+}, a_{-}} p_{n}\left(a_{+}, a_{-}\right)=$ $p_{n}$.

To proceed, define the average positive atmosphere of a polygon of length $n$ by

$$
\left\langle a_{+}\right\rangle_{n}=\frac{\sum_{a_{+}, a_{-}} a_{+} p_{n}\left(a_{+}, a_{-}\right)}{p_{n}} .
$$

Comparison of this with equation (20) shows that

$$
\left\langle a_{+}\right\rangle_{n}=\left\langle a_{-}\right\rangle_{n+2} \frac{p_{n+2}}{p_{n}} .
$$

This may be rearranged to

$$
\frac{p_{n+2}}{p_{n}}=\frac{\left\langle a_{+}\right\rangle_{n}}{\left\langle a_{-}\right\rangle_{n+2}} .
$$

As $n \rightarrow \infty$ this converges to $\mu^{2}$ (see equation (7)). Hence, estimating the ratio of atmospheres on the right hand side for a range of values of $n$, and then extrapolating in $n$, will give an estimate of $\mu^{2}$. 


\begin{tabular}{||c|c|c|c|c||}
\hline$n$ & $\left\langle a_{+} / a_{-}\right\rangle_{n}$ & $\left\langle a_{+}\right\rangle_{n}$ & $\left\langle a_{-}\right\rangle_{n}$ & Ratio \\
\hline 10 & $5.3737 \pm 0.0079$ & $15.3728 \pm 0.0098$ & $3.3932 \pm 0.0048$ & $4.5305 \pm 0.0093$ \\
16 & $7.286 \pm 0.018$ & $23.038 \pm 0.031$ & $4.1385 \pm 0.0077$ & $5.5668 \pm 0.0179$ \\
20 & $8.065 \pm 0.027$ & $28.821 \pm 0.049$ & $4.8638 \pm 0.0110$ & $5.926 \pm 0.024$ \\
30 & $8.654 \pm 0.029$ & $43.513 \pm 0.050$ & $6.9189 \pm 0.0142$ & $6.289 \pm 0.021$ \\
40 & $8.449 \pm 0.026$ & $58.459 \pm 0.051$ & $9.0830 \pm 0.0156$ & $6.4361 \pm 0.0167$ \\
50 & $8.173 \pm 0.023$ & $73.544 \pm 0.055$ & $11.2410 \pm 0.0177$ & $6.5425 \pm 0.0152$ \\
60 & $7.932 \pm 0.021$ & $88.713 \pm 0.064$ & $13.4015 \pm 0.0198$ & $6.6196 \pm 0.0146$ \\
80 & $7.6280 \pm 0.0153$ & $119.014 \pm 0.071$ & $17.725 \pm 0.022$ & $6.7145 \pm 0.0124$ \\
100 & $7.4566 \pm 0.0132$ & $149.376 \pm 0.078$ & $22.094 \pm 0.025$ & $6.7609 \pm 0.0112$ \\
120 & $7.3372 \pm 0.0116$ & $179.590 \pm 0.091$ & $26.494 \pm 0.028$ & $6.7785 \pm 0.0106$ \\
150 & $7.2690 \pm 0.0099$ & $225.207 \pm 0.099$ & $32.940 \pm 0.031$ & $6.8369 \pm 0.0095$ \\
200 & $7.1776 \pm 0.0091$ & $300.817 \pm 0.121$ & $43.887 \pm 0.037$ & $6.8544 \pm 0.0086$ \\
250 & $7.1152 \pm 0.0080$ & $376.339 \pm 0.140$ & $54.834 \pm 0.042$ & $6.8632 \pm 0.0079$ \\
300 & $7.1047 \pm 0.0068$ & $452.416 \pm 0.147$ & $65.627 \pm 0.044$ & $6.8937 \pm 0.0069$ \\
350 & $7.0935 \pm 0.0067$ & $528.410 \pm 0.167$ & $76.401 \pm 0.051$ & $6.9163 \pm 0.0069$ \\
400 & $7.0598 \pm 0.0061$ & $603.730 \pm 0.177$ & $87.424 \pm 0.053$ & $6.9058 \pm 0.0063$ \\
500 & $7.0458 \pm 0.0056$ & $755.49 \pm 0.21$ & $109.128 \pm 0.063$ & $6.9230 \pm 0.0060$ \\
600 & $7.0334 \pm 0.0052$ & $907.12 \pm 0.23$ & $130.852 \pm 0.068$ & $6.9324 \pm 0.0054$ \\
700 & $7.0082 \pm 0.0049$ & $1057.95 \pm 0.25$ & $152.839 \pm 0.077$ & $6.9220 \pm 0.0052$ \\
800 & $7.0103 \pm 0.0046$ & $1209.63 \pm 0.27$ & $174.445 \pm 0.083$ & $6.9342 \pm 0.0049$ \\
900 & $7.0039 \pm 0.0045$ & $1361.13 \pm 0.30$ & $196.200 \pm 0.093$ & $6.9375 \pm 0.0049$ \\
1000 & $6.9970 \pm 0.0034$ & $1512.48 \pm 0.25$ & $218.150 \pm 0.076$ & $6.9332 \pm 0.0036$ \\
1200 & $6.9877 \pm 0.0032$ & $1815.37 \pm 0.29$ & $261.681 \pm 0.086$ & $6.9373 \pm 0.0034$ \\
1500 & $6.9822 \pm 0.0030$ & $2269.94 \pm 0.33$ & $326.991 \pm 0.099$ & $6.9419 \pm 0.0032$ \\
2000 & $6.9794 \pm 0.0026$ & $3027.62 \pm 0.38$ & $435.611 \pm 0.118$ & $6.9503 \pm 0.0028$ \\
2500 & $6.9761 \pm 0.0021$ & $3784.80 \pm 0.39$ & $544.403 \pm 0.117$ & $6.9522 \pm 0.0022$ \\
3000 & $6.97005 \pm 0.00194$ & $4541.54 \pm 0.43$ & $653.443 \pm 0.132$ & $6.9502 \pm 0.0021$ \\
3500 & $6.97188 \pm 0.00181$ & $5299.21 \pm 0.47$ & $761.955 \pm 0.146$ & $6.9548 \pm 0.0020$ \\
4000 & $6.96912 \pm 0.00168$ & $6056.95 \pm 0.51$ & $870.833 \pm 0.153$ & $6.9554 \pm 0.0019$ \\
5000 & $6.96873 \pm 0.00157$ & $7571.18 \pm 0.59$ & $1088.313 \pm 0.179$ & $6.9568 \pm 0.0017$ \\
\hline
\end{tabular}

Table 1. Polygon atmospheres in two dimensions.

Define the average positve atmosphere per edge by $\left\langle a_{+} / n\right\rangle_{n}$ (and similarly for $\left.\left\langle a_{-} / n\right\rangle_{n}\right)$. Kesten's pattern theorem [25, 26] shows that both $\left\langle a_{+} / n\right\rangle_{n}$ and $\left\langle a_{-} / n\right\rangle_{n}$ are bounded as $n \rightarrow \infty$. If one assumes that these averages approach constants as $n \rightarrow \infty$, then equation (23) may be approximated by

$$
\frac{p_{n+2}}{p_{n}} \approx \frac{\left\langle a_{+} / n\right\rangle_{n}}{\left\langle a_{-} / n\right\rangle_{n}}
$$

where the right hand side converges to a constant as $n \rightarrow \infty$ in which case the approximation becomes exact.

The averages $\left\langle a_{ \pm} / n\right\rangle=\left\langle a_{ \pm}\right\rangle / n$ can be collected using the pivot algorithm for polygons at fixed values of $n$ [28]. Since each atmosphere is computed by examining each edge of a polygon, it follows that atmospheres are typically $O(n d)$ in a $d$ dimensional polygon of length $n$. In constrast, the atmospheres defined for the selfavoiding walk in reference $[40,20]$ are $O(d)$. 


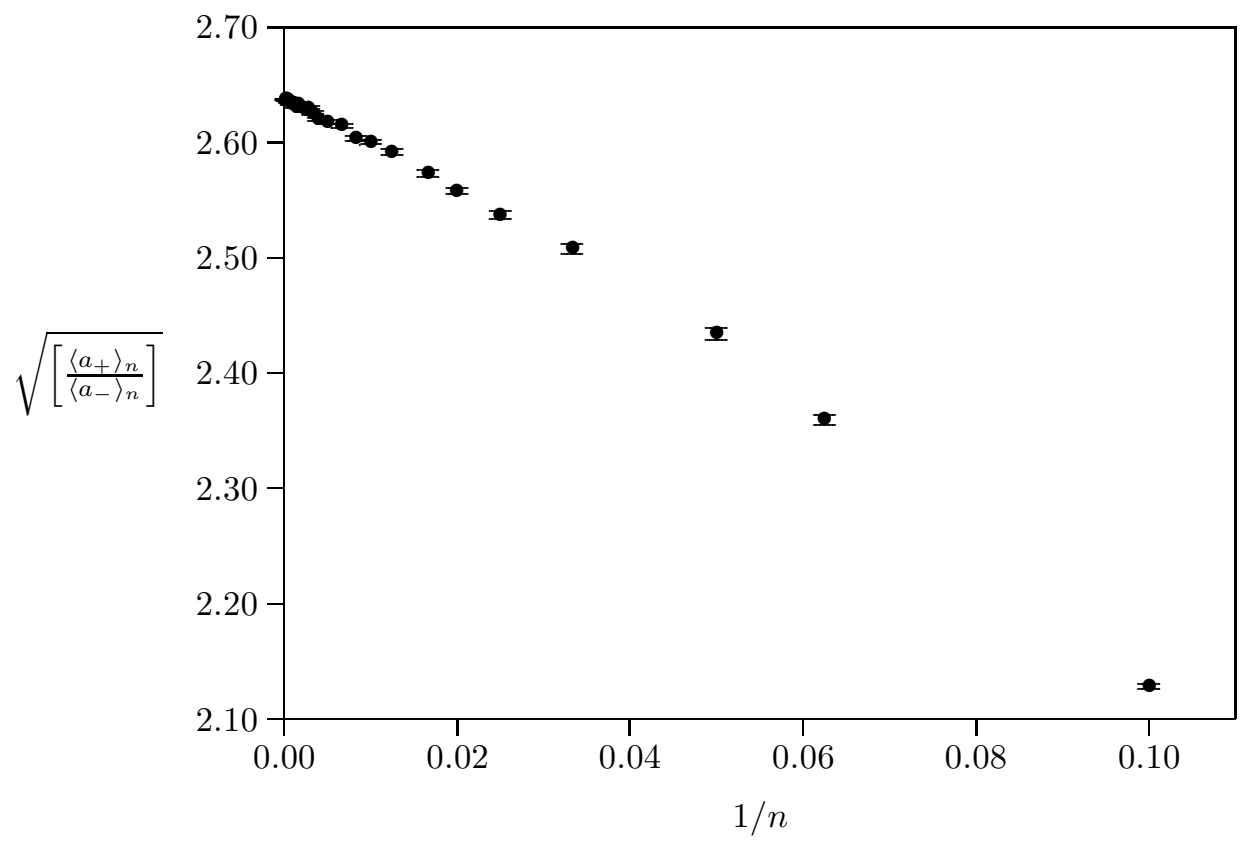

Figure 4. The ratio $\left[\left\langle a_{+}\right\rangle_{n} /\left\langle a_{-}\right\rangle_{n}\right]^{1 / 2}$ plotted against $1 / n$ in the square lattice. Extrapolation of the data to infinite $n$ is done by a regression with the model $2 \mathrm{~d}-1$ to estimate $\mu$.

\section{Connective constants of polygons on the square and cubic lattice}

The pivot algorithm for polygons [28, 22] was used to collect the atmospheric data in two dimensions (see Table 1) and in three dimensions (see Table 4). The lengths of polygons was set at values between 10 and 5000, and for each value of $n$ (except $n=700$ and $n=5000$ ), the number of iterations was set at $N \times 10^{5} n$ with data collected every $\lceil n / 10\rceil$ iterations. The value of $N$ was increased from $N=2$ for $n=10$ to $N=4$ for $n=5000$.

\subsection{Results in 2 dimensions}

Data collected in two dimensions are tabulated in Table 1 . The ratios of the atmospheres were also collected, and these are plotted in figure 4 against $1 / n$. It appears that the ratio of the atmospheres quickly approaches $\mu^{2}$ with increasing $n$.

The data in Table 1 were analysed using weighted least squares. Assuming that $p_{n}=A n^{\alpha-2} \mu^{n}(1+o(1))$ suggests fitting the data to the model

$$
\left.\frac{\left\langle a_{+}\right\rangle_{n}}{\left\langle a_{-}\right\rangle_{n}}=\mu^{2}((n+2) / n)\right)^{a_{2}}
$$

where $a_{2}$ is a constant. One would expect that $a_{2}=\alpha-2$. This model requires a non-linear least squares analysis and did not behave well numerically.

Instead, we took logarithms on both sides of the last equation to get the model

$$
\left.\log \left(\frac{\left\langle a_{+}\right\rangle_{n}}{\left\langle a_{-}\right\rangle_{n}}\right)=\log \mu^{2}+a_{2} \log ((n+2) / n)\right)+a_{3} / n^{2}, \quad(\text { model } 2 \mathrm{~d}-1)
$$




\begin{tabular}{||c|c|c|c|c||}
\hline$n_{\min }$ & $\log \mu^{2}$ & $a_{2}$ & $\chi^{2}(\mathrm{df})$ & Level \\
\hline 10 & $1.939904 \pm 0.000078$ & $-1.323 \pm 0.019$ & $49.0(26)$ & $99.6 \%$ \\
16 & $1.940015 \pm 0.000081$ & $-1.406 \pm 0.025$ & $35.2(25)$ & $91.4 \%$ \\
20 & $1.940081 \pm 0.000083$ & $-1.460 \pm 0.029$ & $28.1(24)$ & $74.4 \%$ \\
30 & $1.940092 \pm 0.000087$ & $-1.470 \pm 0.036$ & $28.1(23)$ & $78.5 \%$ \\
40 & $1.940065 \pm 0.000090$ & $-1.444 \pm 0.043$ & $27.4(22)$ & $80.2 \%$ \\
50 & $1.940078 \pm 0.000093$ & $-1.458 \pm 0.049$ & $27.2(21)$ & $83.6 \%$ \\
\hline
\end{tabular}

Table 2. Least squares analysis results: model $2 \mathrm{~d}-1$.

where one extra parameter $a_{3}$ was inserted to control for deviations in the data for small values of $n$. Observe that $\log ((n+2) / n)=1 / n+O\left(1 / n^{2}\right)$ so that this is a linear model in $1 / n$.

Linear least squares analysis of the data in Table 1 using model 2d-1 (equation (26)) gave acceptable fits: we tracked the weighted least square error $\chi^{2}\left(n_{\min }\right)$ as a function of the smallest value of $n\left(n_{m i n}\right)$ included in the analysis. This statistic is distributed as a $\chi^{2}$-statistic on $d f$ degrees of freedom, where $d f$ is the number of datapoints minus the number of parameters in the model. A fit is statistically acceptable if the least square error is acceptable at the $95 \%$ level.

The results of the analysis are given in Table 2 . For $n_{\min }=16$ the fit is acceptable at the $91.4 \%$ level. In this case $\log \mu^{2}=1.940015 \pm 0.000081$ ( $67 \%$ statistical confidence interval). Increasing $n_{\min }$ first to 20 and then in steps to $n_{\min }=50$ gave better fits. For $n_{\text {min }}>10$ all the regressions gave a value of $\log \mu^{2}$ within the statistical confidence interval of the regression at $n_{\min }=16$, but the value of $\log \mu^{2}$ for larger values of $n_{\min }$ settled down to a stable value within the confidence interval of its value at $n_{\min }=20$. We took the result at $n_{\min }$ as our best estimate for $\mu$ : Dividing by 2 and exponentiating gives

$$
\mu=2.63805 \pm 0.00011
$$

with a $67 \%$ statistical confidence interval.

The value of the parameter $a_{2}$ in model (26) is consistently in the vicinity of -1.5 ; this agrees with the expectation that $a_{2}=\alpha-2=-3 / 2$. With increasing $n_{\text {min }}$ the estimate settles on a value that includes $-3 / 2$ well inside its confidence interval.

Estimates of the ratio $a_{+} / a_{-}$were also collected in our simulations. Since this ratio is undefined if $a_{-}=0$, we modified $a_{-}$by putting $a_{-}=1$ whenever the negative

\begin{tabular}{||c|c|c|c|c||}
\hline$n_{\min }$ & $\log \mu^{2}$ & $a_{2}$ & $\chi^{2}(\mathrm{df})$ & Level \\
\hline 10 & $1.938586 \pm 0.000072$ & $4.245 \pm 0.018$ & $1600(26)$ & $100 \%$ \\
16 & $1.938108 \pm 0.000076$ & $4.614 \pm 0.025$ & $1379(25)$ & $100 \%$ \\
20 & $1.938544 \pm 0.000077$ & $4.242 \pm 0.029$ & $1012(24)$ & $100 \%$ \\
30 & $1.939493 \pm 0.000081$ & $3.375 \pm 0.037$ & $236(23)$ & $100 \%$ \\
40 & $1.939925 \pm 0.000084$ & $2.951 \pm 0.043$ & $56.4(22)$ & $100 \%$ \\
50 & $1.940094 \pm 0.000087$ & $2.775 \pm 0.049$ & $27.3(21)$ & $83.9 \%$ \\
60 & $1.940138 \pm 0.000090$ & $2.726 \pm 0.055$ & $25.5(20)$ & $81.8 \%$ \\
80 & $1.940137 \pm 0.000095$ & $2.727 \pm 0.065$ & $25.5(19)$ & $85.6 \%$ \\
\hline
\end{tabular}

Table 3. Least squares analysis results: model $2 \mathrm{~d}-2$. 


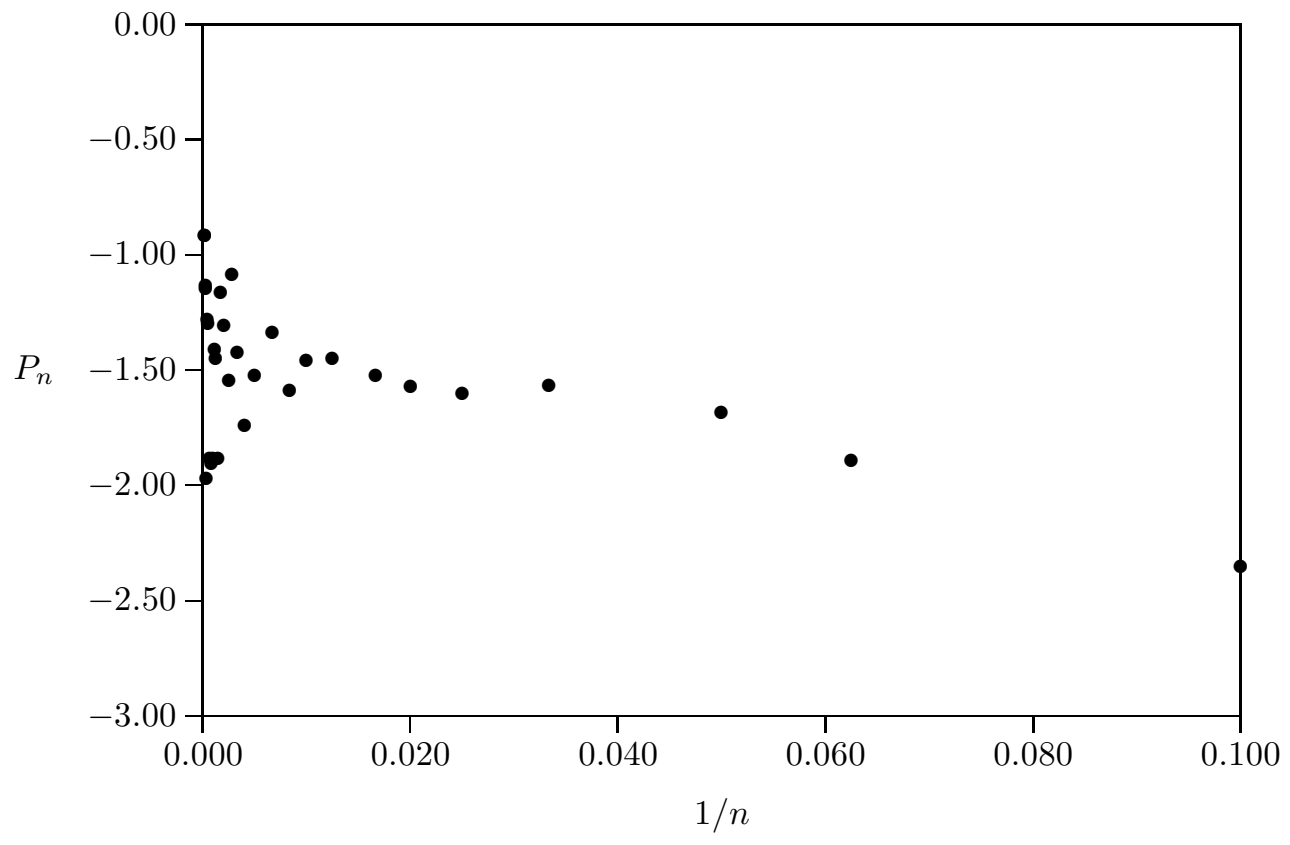

Figure 5. Scatterplot of $P_{n}=n\left(\log \left(\frac{\left\langle a_{+}\right\rangle}{\langle a+\rangle}\right)-\log \mu^{2}\right)$ against $1 / n$. Error bars have been left away for clarity. $\mu^{2}$ was set equal to its least squares value in equation (30). The data points accummulate around -1.5 ; this is consistent with $\alpha=1 / 2$.

atmosphere of a polygon is zero. Since the probability that a polygon will have no negative atmospheric squares goes to zero quickly with increasing $n$, this slight modification is only relevent at small values of $n$, and consequently did not disturb the average at higher values of $n$. Our estimates are also listed in Table 1. These data were similarly analysed using the three parameter model

$$
\left.\log \left\langle\frac{a_{+}}{a_{-}}\right\rangle_{n}=\log \mu^{2}+a_{2} \log ((n+2) / n)\right)+a_{3} / n^{2}, \quad(\text { model } 2 \mathrm{~d}-2) .
$$

The results of the regression is given in Table 3. Again, the least square error was tracked as a function of $n_{\min }$. The regressions only became statistically acceptable at the $95 \%$ level for $n_{\min }=40$, and good fits were obtained for $n_{\min }=50$ and higher. By taking the results at $n_{\min }=50$ as our best estimates, we get

$$
\mu=2.63807 \pm 0.00012
$$

with a $67 \%$ statistical confidence interval. This demonstrates that the statistic $\left(a_{+} / a_{-}\right)$can also be tracked and analysed to obtain estimates for $\mu$.

There are no a priori reasons to accept any one of the above results as our best estimate, so we averaged them and took the largest confidence interval as an error bar:

$$
\mu=2.63806 \pm 0.00012 .
$$

This result is consistent with the estimates in equations (8), (10), (16) and (17). 
The exponent $\alpha$ in equation (6) can also be estimated from our data. By equation (26) we observe that

$$
\left.\left(\log \left(\frac{\left\langle a_{+}\right\rangle_{n}}{\left\langle a_{-}\right\rangle_{n}}\right)-\log \mu^{2}\right)=a_{2} \log ((n+2) / n)\right)+O(1 / n),
$$

and hence by plotting $\left(\log \left(\frac{\left\langle a_{+}\right\rangle_{n}}{\left\langle a_{-}\right\rangle_{n}}\right)-\log \mu^{2}\right) / \log ((n+2) / n)$ against $1 / n$, the $Y$ intercept should be $a_{2}=\alpha-2$ as $1 / n \rightarrow 0^{+}$. These data are plotted in Figure 5 as a scatter plot for clarity. The data points accummulate close to -1.5 ; this is consistent with the expectation that $\alpha=1 / 2$ in this model. A weighted least squares fit to the data in Figure 5 using the model

$$
n\left(\log \left(\frac{\left\langle a_{+}\right\rangle}{\left\langle a_{+}\right\rangle}\right)-\log \mu^{2}\right)=(\alpha-2)+\frac{a 1}{n}+\frac{a 2}{n^{2}}
$$

gives a statistically acceptable regression at the $78 \%$ level. The estimate for the specific heat exponent is $2-\alpha=-1.468 \pm 0.027$ with a $67 \%$ confidence interval. Thus, we estimate $\alpha=0.532 \pm 0.027$. Hyperscaling in models of self-avoiding walks is exhibited in the scaling relation $2-\alpha=1 / \phi=d \nu$ in $d$ dimensions, where $\nu$ is the metric exponent and $\phi$ is the crossover exponent. Conformal field theory and Coulomb gas techniques shows that the exact value for the metric exponent is $\nu=3 / 4$, and thus $\phi=2 / 3$ and $\alpha=1 / 2$ by hyperscaling [33,34]. These values are consistent with the numerical estimate of $\alpha$ above, which includes $1 / 2$ within its $95 \%$ confidence interval.

\subsection{Results in 3 dimensions}

We performed similar simulations and calculations in three dimensions. The data collected in three dimensions are displayed in Table 4.

The model

$$
\left.\log \left(\frac{\left\langle a_{+}\right\rangle_{n}}{\left\langle a_{-}\right\rangle_{n}}\right)=\log \mu^{2}+a_{2} \log ((n+2) / n)\right)+a_{3} / n^{2}, \quad(\text { model 3d-1) }
$$

was examined for the ratios of the atmospheric data in Table 4. Regression data for this model are listed in Table 5. Even for $n_{\min }=10$ the regression is acceptable at the $95 \%$ level on 26 degrees of freedom. In this case, the best estimate obtained for $\mu$ is

$$
\mu=4.683644 \pm 0.000037 \text {. }
$$

Increasing the value of $n_{\text {min }}$ to 20 gave $4.683867 \pm 0.000038$, which is outside the error bar at $n_{\min }=10$. Further increases in $n_{\min }$ did not cause further significant changes in the estimate, and we take the value at $n_{\min }=20$ to be our best estimate:

$$
\mu=4.683867 \pm 0.000038 \text {. }
$$

The model

$$
\left.\log \left\langle\frac{a_{+}}{a_{-}}\right\rangle_{n}=\log \mu^{2}+a_{2} \log ((n+2) / n)\right)+a_{3} / n^{2}, \quad \text { (model 3d-2). }
$$

was also examined. Regression data is given in Table 6 . The fits were not acceptable for small values of $n_{\min }$, but the quality of the regressions increased with increasing $n_{\min }$ and regressions acceptable at the $95 \%$ level were obtained for $n_{\min } \geq 50$. In this case the best estimate obtained for $\mu$ is

$$
\mu=4.683972 \pm 0.000041 \text {. }
$$




\begin{tabular}{||c|c|c|c|c|}
\hline$n$ & $\left\langle a_{+} / a_{-}\right\rangle$ & $\left\langle a_{+}\right\rangle$ & $\left\langle a_{-}\right\rangle$ & Ratio \\
\hline 10 & $15.7918 \pm 0.0171$ & $45.4003 \pm 0.0146$ & $3.2247 \pm 0.0046$ & $14.079 \pm 0.025$ \\
16 & $21.476 \pm 0.028$ & $70.117 \pm 0.023$ & $4.0795 \pm 0.0055$ & $17.188 \pm 0.029$ \\
20 & $23.512 \pm 0.038$ & $87.117 \pm 0.031$ & $4.7959 \pm 0.0069$ & $18.165 \pm 0.033$ \\
30 & $25.370 \pm 0.044$ & $130.611 \pm 0.040$ & $6.7335 \pm 0.0085$ & $19.397 \pm 0.031$ \\
40 & $25.339 \pm 0.042$ & $174.729 \pm 0.048$ & $8.7046 \pm 0.0096$ & $20.073 \pm 0.028$ \\
50 & $24.723 \pm 0.039$ & $219.059 \pm 0.055$ & $10.7366 \pm 0.0110$ & $20.403 \pm 0.027$ \\
60 & $24.224 \pm 0.035$ & $263.598 \pm 0.059$ & $12.7390 \pm 0.0120$ & $20.692 \pm 0.025$ \\
80 & $23.458 \pm 0.028$ & $352.742 \pm 0.069$ & $16.8055 \pm 0.0142$ & $20.990 \pm 0.022$ \\
100 & $23.077 \pm 0.024$ & $442.079 \pm 0.079$ & $20.8720 \pm 0.0163$ & $21.180 \pm 0.021$ \\
120 & $22.862 \pm 0.021$ & $531.484 \pm 0.089$ & $24.9350 \pm 0.0184$ & $21.315 \pm 0.020$ \\
150 & $22.6420 \pm 0.0184$ & $665.734 \pm 0.096$ & $31.049 \pm 0.021$ & $21.4414 \pm 0.0176$ \\
200 & $22.4215 \pm 0.0163$ & $889.395 \pm 0.116$ & $41.285 \pm 0.026$ & $21.5428 \pm 0.0164$ \\
250 & $22.3361 \pm 0.0144$ & $1113.621 \pm 0.134$ & $51.457 \pm 0.028$ & $21.6418 \pm 0.0143$ \\
300 & $22.2695 \pm 0.0138$ & $1337.490 \pm 0.153$ & $61.650 \pm 0.032$ & $21.6949 \pm 0.0138$ \\
350 & $22.2022 \pm 0.0125$ & $1561.350 \pm 0.167$ & $71.914 \pm 0.035$ & $21.7113 \pm 0.0129$ \\
400 & $22.1758 \pm 0.0118$ & $1785.610 \pm 0.181$ & $82.100 \pm 0.037$ & $21.7492 \pm 0.0121$ \\
500 & $22.1269 \pm 0.0105$ & $2233.66 \pm 0.21$ & $102.529 \pm 0.042$ & $21.7856 \pm 0.0110$ \\
600 & $22.0821 \pm 0.0099$ & $2682.08 \pm 0.23$ & $123.025 \pm 0.048$ & $21.8011 \pm 0.0104$ \\
700 & $22.0839 \pm 0.0093$ & $3130.35 \pm 0.25$ & $143.325 \pm 0.053$ & $21.8409 \pm 0.0099$ \\
800 & $22.0469 \pm 0.0090$ & $3578.04 \pm 0.28$ & $163.868 \pm 0.058$ & $21.8349 \pm 0.0095$ \\
900 & $22.0442 \pm 0.0088$ & $4026.85 \pm 0.31$ & $184.236 \pm 0.064$ & $21.8570 \pm 0.0093$ \\
1000 & $22.0312 \pm 0.0066$ & $4474.69 \pm 0.26$ & $204.668 \pm 0.053$ & $21.8632 \pm 0.0070$ \\
1200 & $22.0170 \pm 0.0062$ & $5371.08 \pm 0.30$ & $245.510 \pm 0.060$ & $21.8772 \pm 0.0066$ \\
1500 & $21.9997 \pm 0.0056$ & $6715.97 \pm 0.33$ & $306.839 \pm 0.068$ & $21.8876 \pm 0.0060$ \\
2000 & $21.9897 \pm 0.0050$ & $8957.19 \pm 0.39$ & $408.922 \pm 0.081$ & $21.9044 \pm 0.0053$ \\
2500 & $21.9753 \pm 0.0040$ & $11198.70 \pm 0.39$ & $511.163 \pm 0.080$ & $21.9083 \pm 0.0042$ \\
3000 & $21.9696 \pm 0.0037$ & $13439.49 \pm 0.44$ & $613.286 \pm 0.091$ & $21.9139 \pm 0.0040$ \\
3500 & $21.9627 \pm 0.0036$ & $15680.75 \pm 0.48$ & $715.523 \pm 0.101$ & $21.9151 \pm 0.0038$ \\
4000 & $21.9652 \pm 0.0033$ & $17923.15 \pm 0.52$ & $817.535 \pm 0.111$ & $21.9234 \pm 0.0037$ \\
5000 & $21.9545 \pm 0.0031$ & $22404.56 \pm 0.59$ & $1022.050 \pm 0.125$ & $21.9212 \pm 0.0033$ \\
\hline & & & &
\end{tabular}

Table 4. Atmospheres in Three Dimensions.

Further increases in $n_{\min }$ to 60 gave $4.684092 \pm 0.000042$, which is outside the error bar at $n_{\min }=50$. Further increases in $n_{\min }$ did not cause further significant changes in this estimate, and we took the value at $n_{\min }=60$ to be our best estimate:

$$
\mu=4.684092 \pm 0.000042 \text {. }
$$

There are no a priori reasons to accept any one of the above results as our best estimate, so we averaged them and took the largest confidence interval as an error bar:

$$
\mu=4.683980 \pm 0.000042 \text {. }
$$

This result compares well with the estimate by Clisby, Liang and Slade [5] in equation (12).

As was the case in two dimensions, our data are consistent with the exponent $\alpha$ in equation (6). By plotting $\left(\log \left(\frac{\left\langle a_{+}\right\rangle_{n}}{\left\langle a_{-}\right\rangle_{n}}\right)-\log \mu^{2}\right) / \log ((n+2) / n)$ against $1 / n$, and 


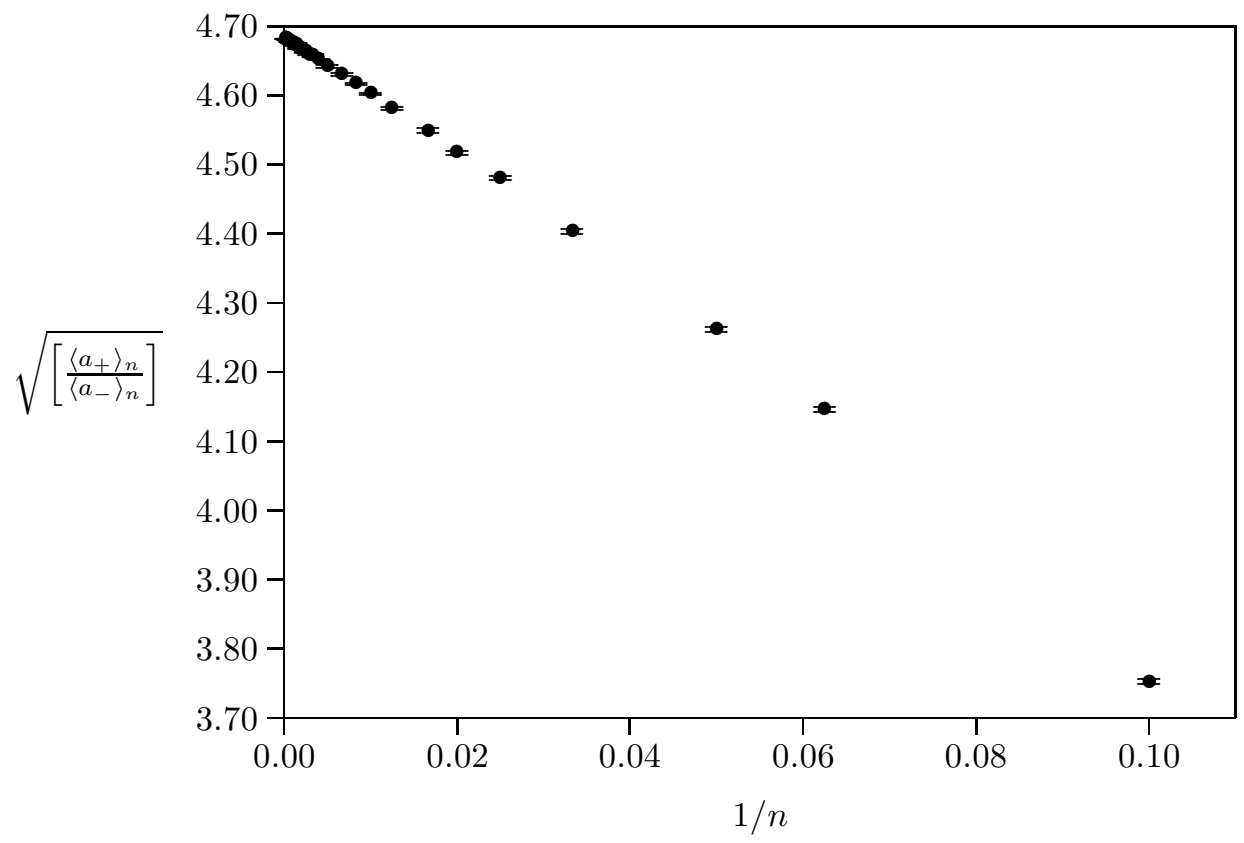

Figure 6. The ratio $\left[\left\langle a_{+}\right\rangle_{n} /\left\langle a_{-}\right\rangle_{n}\right]^{1 / 2}$ plotted against $1 / n$ in the cubic lattice. Extrapolation of the data to infinite $n$ is done by a regression with the model $3 \mathrm{~d}-1$ to estimate $\mu$.

by considering equation (33), it follows that the $Y$-intercept should be at $a_{2}=\alpha-2$ as $1 / n \rightarrow 0^{+}$. The data are plotted in Figure 7 as a scatter plot.

The data points accummulate close to -1.75 ; this is consistent with the expectation that $\alpha \approx 0.25$ in this model. A weighted least squares fit to the data in Figure 5 using the model

$$
n\left(\log \left(\frac{\left\langle a_{+}\right\rangle}{\left\langle a_{+}\right\rangle}\right)-\log \mu^{2}\right)=(\alpha-2)+\frac{a 1}{n}+\frac{a 2}{n^{2}}
$$

gives a statistically acceptable regression. The estimate for the specific heat exponent is $2-\alpha=-1.7525 \pm 0.0151$ with a $67 \%$ confidence interval. Increasing $n_{\min }$ in the fit did not change this estimate outside its confidence intervals, and so our best estimate is $\alpha=0.248 \pm 0.016$. These results are consistent with the estimates in reference [5] where it is estimated that $\alpha$ is in the range 0.23 to 0.24 .

\begin{tabular}{||c|c|c|c|c||}
\hline$n_{\min }$ & $\log \mu^{2}$ & $a_{2}$ & $\chi^{2}(\mathrm{df})$ & Level \\
\hline 10 & $3.088153 \pm 0.000048$ & $-1.654 \pm 0.0192$ & $33.4(26)$ & $85 \%$ \\
16 & $3.088237 \pm 0.000049$ & $-1.714 \pm 0.0136$ & $11.3(25)$ & $0.8 \%$ \\
20 & $3.088248 \pm 0.000050$ & $-1.723 \pm 0.0158$ & $10.7(24)$ & $0.9 \%$ \\
30 & $3.088244 \pm 0.000053$ & $-1.720 \pm 0.021$ & $10.7(23)$ & $1.4 \%$ \\
40 & $3.088263 \pm 0.000055$ & $-1.737 \pm 0.025$ & $9.9(22)$ & $1.2 \%$ \\
\hline
\end{tabular}

Table 5. Least squares analysis results: model 3d-1. 


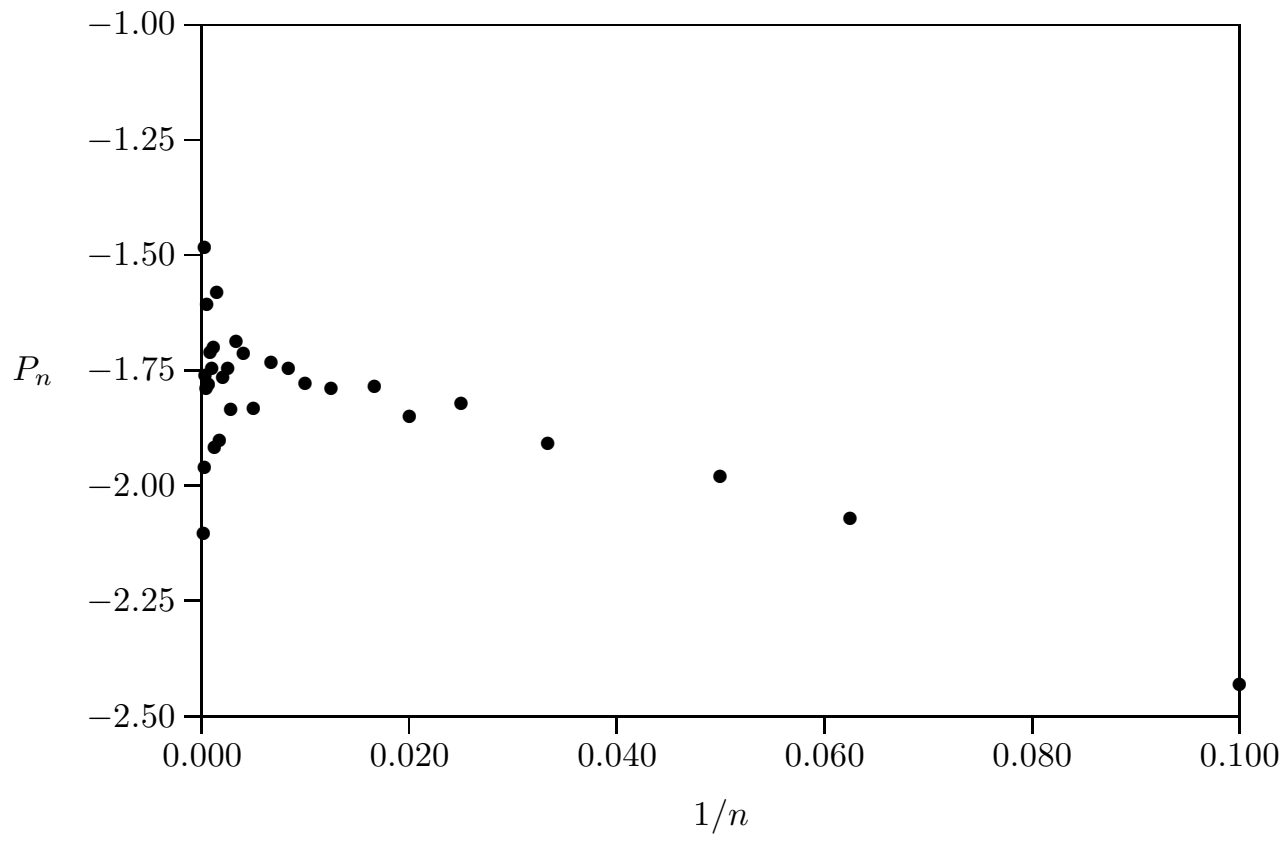

Figure 7. Scatterplot of $P_{n}=n\left(\frac{\left\langle a_{+}\right\rangle}{\left\langle a_{+}\right\rangle}-\log \mu^{2}\right)$ against $1 / n$. Error bars have been removed for clarity. $\mu^{2}$ was set equal to its least squares value in equation (39). The data points accummulate around -1.77 ; this is consistent with $\alpha \approx 0.23$.

\section{Relative Free Energy of Knotted Polygons}

In this section we turn our attention to polygons in the cubic lattice with fixed knot type. Knotted polygons have received attention in the literature [31, 32, 41, 38] as models of knotted polymers.

We define $p_{n}(K)$ to be the number of lattice polygons of length $n$ and with knot type $K$, (counted up to translations in the lattice). The atmospheres of a polygon of fixed knot type are defined as in section 2 , and following the arguments leading up to

\begin{tabular}{||c|c|c|c|c||}
\hline$n_{\min }$ & $\log \mu^{2}$ & $a_{2}$ & $\chi^{2}(\mathrm{df})$ & Level \\
\hline 10 & $3.087069 \pm 0.000044$ & $3.2151 \pm 0.0099$ & $3459(26)$ & $100 \%$ \\
16 & $3.086527 \pm 0.000046$ & $3.6196 \pm 0.0142$ & $2678(25)$ & $100 \%$ \\
20 & $3.086816 \pm 0.000047$ & $3.3815 \pm 0.0163$ & $2239(24)$ & $100 \%$ \\
30 & $3.087665 \pm 0.000050$ & $2.631 \pm 0.022$ & $640(23)$ & $100 \%$ \\
40 & $3.088140 \pm 0.000052$ & $2.176 \pm 0.026$ & $80.5(22)$ & $100 \%$ \\
50 & $3.088293 \pm 0.000054$ & $2.019 \pm 0.029$ & $18.3(21)$ & $36.7 \%$ \\
60 & $3.088344 \pm 0.000055$ & $1.964 \pm 0.033$ & $11.8(20)$ & $7.6 \%$ \\
80 & $3.088317 \pm 0.000059$ & $1.994 \pm 0.039$ & $10.8(20)$ & $6.8 \%$ \\
\hline
\end{tabular}

Table 6. Least squares analysis results: model $3 \mathrm{~d}-2$. 
equation (23), it follows that

$$
\frac{p_{n+2}(K)}{p_{n}(K)}=\frac{\left\langle a_{+}(K)\right\rangle_{n}}{\left\langle a_{-}(K)\right\rangle_{n+2}} .
$$

Observe that the knot type of the polygon is knot changed by adding edges around positive atmospheric squares, or by removing edges around negative atmospheric squares.

There is little known about $p_{n}(K)$. In particular, it is normally assumed that

$$
p_{n}(K) \approx C_{K} n^{\left(\alpha_{K}-2\right)}\left(\mu_{K}\right)^{n}
$$

where $\mu_{K}$ is a growth constant, $\alpha_{K}$ is an entropic exponent of polygons of knot type $K$, and $C_{K}$ is a constant. Similations indicate that $\alpha_{K}=\alpha_{\emptyset}+N_{K}$ where $N_{K}$ is the number of prime components in the knot $K$ and that $\alpha_{\emptyset}=\alpha$, where $\alpha_{\emptyset}$ is the entropic exponent of unknotted polygons, and $\alpha \approx 0.25$ is the entropic exponent of polygons in the cubic lattice [37].

We define the entropy of polygons of length $n$ and knot type $K$ by

$$
E_{n}(K)=\log p_{n}(K)
$$

and the relative entropy is then given from equation (41) by

$$
E_{n+2}(K)-E_{n}(K)=\log \left\langle a_{+}(K)\right\rangle_{n}-\log \left\langle a_{-}(K)\right\rangle_{n+2} .
$$

Kesten's pattern theorem for polygons (see reference [41]) suggests that $\left\langle a_{+}(K)\right\rangle_{n} / n \rightarrow$ $\alpha_{+}$and $\left\langle a_{-}(K)\right\rangle_{n} / \rightarrow \alpha_{-}$as $n \rightarrow \infty$, where $\alpha_{+}$and $\alpha_{-}$are constants. Taking $n$ to infinity in the last equation shows that

$$
\lim _{n \rightarrow \infty}\left(E_{n+2}(K)-E_{n}(K)\right)=\log \left(\alpha_{+} / \alpha_{-}\right) .
$$

In other words, a plot of $\left[p_{n+2}(K) / p_{n}(K)\right]^{1 / 2}$ against $n$ will approach the ratio $\left(\alpha_{+} / \alpha_{-}\right)^{1 / 2}$. Such a plot is given in Figure 8 for $K=\emptyset$, the unknot.

The entropy of knotted polygons of knot type $K$ is given by

$$
E_{n}(K)=\sum_{m=n_{m i n}}^{n-2}\left(E_{m+2}(K)-E_{m}(K)\right)+E_{n_{m i n}}(K)
$$

where $n_{\min }$ is the minimum number of edges necessary to realise a polygon of knot type $K$ in the cubic lattice [7, 19] (this is the minimal edge number of the knot type). This shows that

$$
E_{n}(K)=\sum_{m=n_{\text {min }}}^{n-2}\left[\log \left(\frac{\left\langle a_{+}(K)\right\rangle_{n}}{\left\langle a_{-}(K)\right\rangle_{n+2}}\right)\right]+E_{n_{\text {min }}}(K) .
$$

The absolute entropy of knotted polygons for $n=n_{\min }$ is an issue which have been addressed for polygons of knot type $3_{1}$ (trefoils) by Diao [8]. In this case, $p_{24}\left(3_{1}\right)=3496$ since $n_{\min }=24$ for trefoils, and thus the entropy is $E_{24}\left(3_{1}\right)=\log 3496$. By computing the area under the curve $\log \left(\left\langle a_{+}(K)\right\rangle_{n} /\left\langle a_{-}(K)\right\rangle_{n+2}\right)$ and adding the entropy at $n_{\text {min }}$, the entropy of knotted polygons at larger values of $n$ can be determined. We did not follow this approach, but instead focussed on determining the relative entropies of knotted polygons.

Unknotted polygons were sampled in the grand canonical ensemble by using the BFACF algorithm [1, 2] which is known to be ergodic for polygons of fixed knot types in the cubic lattice $[18,21]$. Polygons of fixed knot type $K$ were sampled along a 


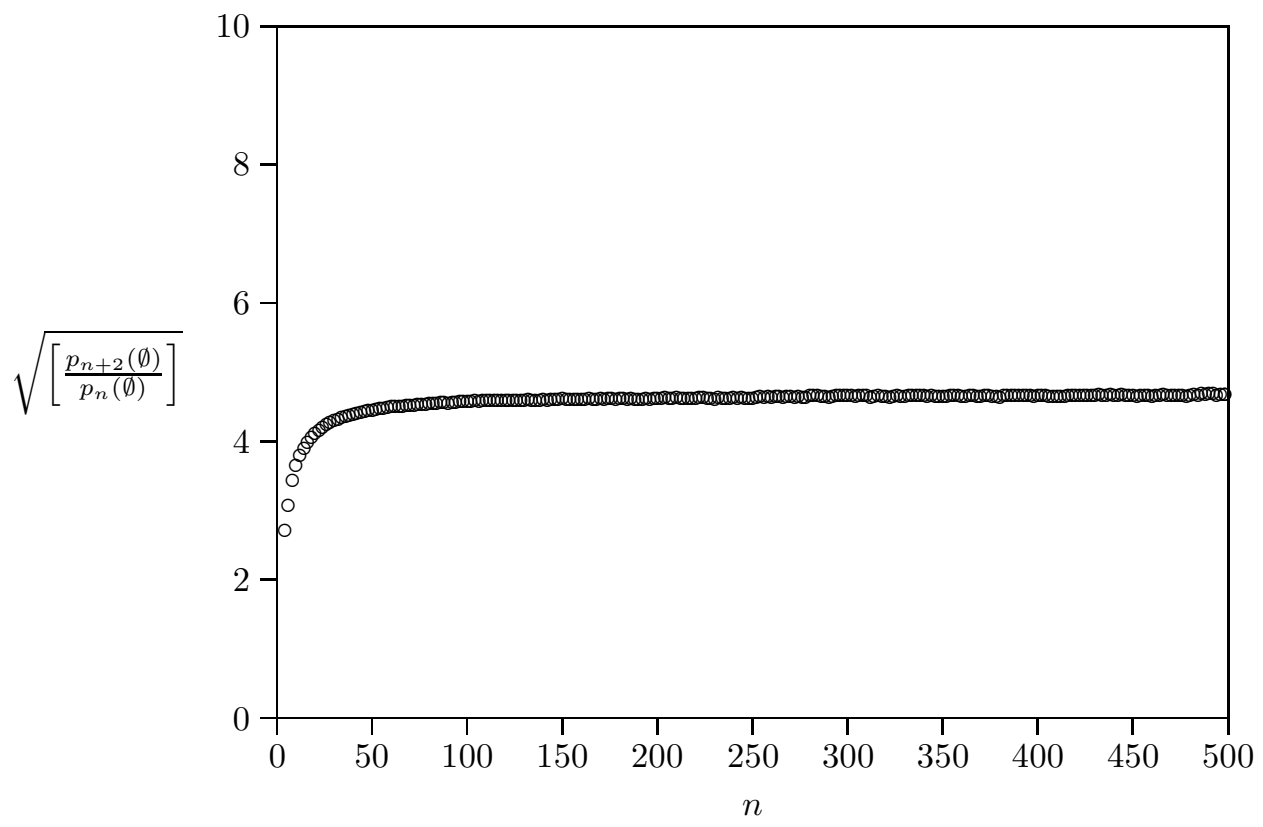

Figure 8. The ratio $\left[p_{n+2}(\emptyset) / p_{n}(\emptyset)\right]^{1 / 2}$ against $n$ for unknotted polygons. Note that the data appears to be monotonically increasing to a limiting value.

Markov Chain for $2 \times 10^{9}$ iterations with data collected every 200 iterations for a time-series of length $10^{7}$ of positive and negative atmospheres.

The data along the time series were binned according to length, and then were analysed at each fixed length to estimate $\left\langle a_{+}(K)\right\rangle_{n}$ and $\left\langle a_{-}(K)\right\rangle_{n}$. Throughout the simulations, the parameter of the BFACF algorithm was set close to its critical value to obtain enough data at large values of $n$ (up to $n=500$ ). These calculations did not produce uncorrelated data at neighbouring values of $n$, but statistical error bars at any given value of $n$ were small, and plots of the data show reasonably smooth curves.

In the case of unknotted polygons (see Figure 8) it is known that the limit

$$
\mu_{\emptyset}=\lim _{n \rightarrow \infty}\left[p_{n}(\emptyset)\right]^{1 / n}
$$

exists via a concatenation argument [41]. Thus, one may expect that

$$
\lim _{n \rightarrow \infty}\left[\frac{p_{n+2}(\emptyset)}{p_{n}(\emptyset)}\right]=\mu_{\emptyset}^{2} .
$$

This remains an open question, even though Figure 8 provides strong evidence in its favour. While the data in Figure 8 quickly settles down close to $\mu_{\emptyset}$ for (say) $n>50$, it is an increasing curve indicating that relative entropy (equation (44)) increases steeply with $n$ for small values of $n$.

In Figure 9 data for the trefoil knot is plotted. The limit

$$
\mu_{3_{1}}=\limsup _{n \rightarrow \infty}\left[p_{n}\left(3_{1}\right)\right]^{1 / n}
$$

is not known to exist, but we give strong numerical data in Figure 9 that

$$
\limsup _{n \rightarrow \infty}\left[\frac{p_{n+2}\left(3_{1}\right)}{p_{n}\left(3_{1}\right)}\right]=\mu_{3_{1}}^{2}
$$




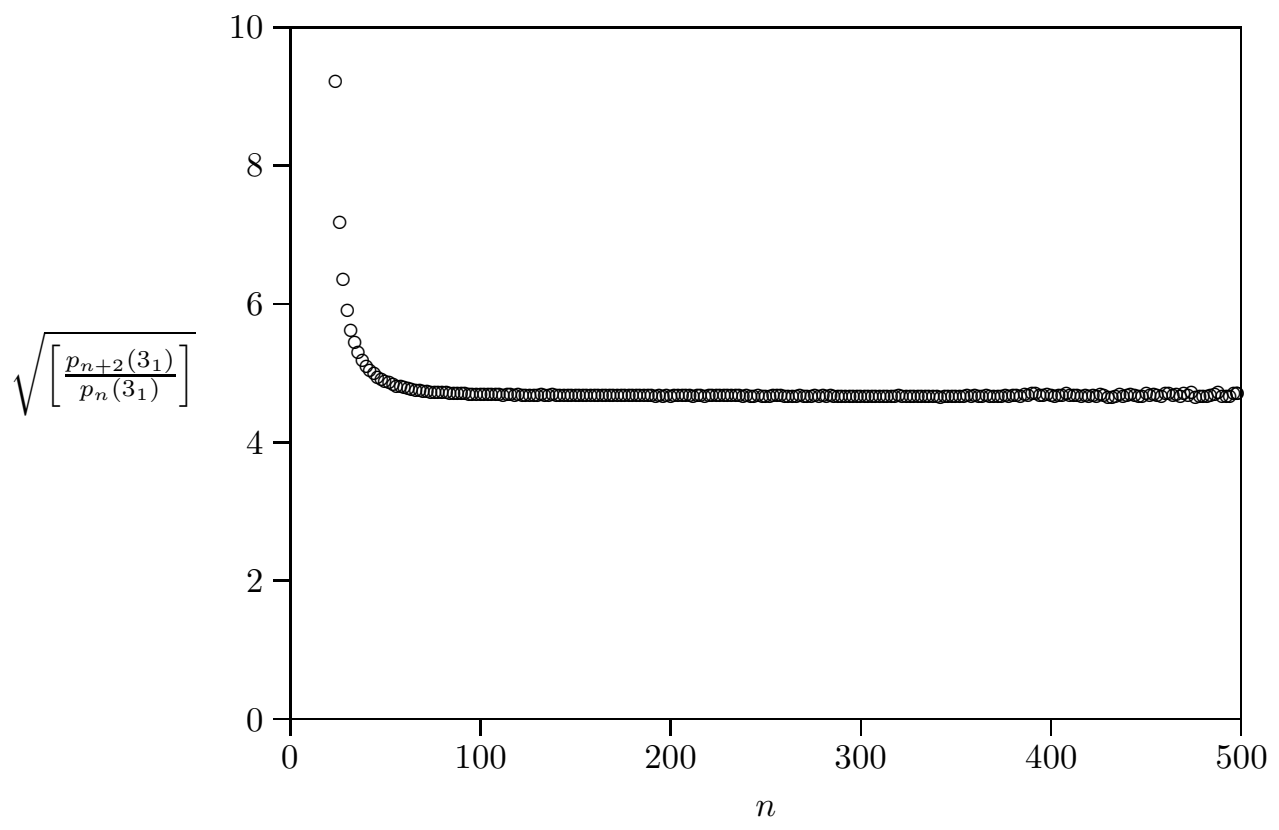

Figure 9. The ratio $\left[p_{n+2}\left(3_{1}\right) / p_{n}\left(3_{1}\right)\right]^{1 / 2}$ against $n$ for polygons with knot type the trefoil. In contrast with the data in Figure 8, the statistic appears to be monotonically decreasing with $n$. Observe that $n \geq 24$, since the polygons with knot type $3_{1}$ have length at least 24 edges.

exists as a limit. In addition, the data suggest that any difference in the values of $\mu_{\emptyset}$ and $\mu_{3_{1}}$ will be small. This is consistent with previous data suggesting that $\mu_{\emptyset}=\mu_{3_{1}}$ [37]. Figure 9 also show that the relative entropy of polygons with knot type $3_{1}$ decreases with increasing $n$. This is the opposite to behaviour for the unknot in Figure 8, and it seems likely that the limit (51) approaches $\mu_{3_{1}}^{2}$ from above, while the limit (49) approaches $\mu_{\emptyset}^{2}$ from below.

In Figure 10, curves are plotted for the unknot and for compounded trefoils against $n$. The starting points of the curves increases with the number of knot components in each knotted polygon, since the number edges required to realize the knots, $n_{\text {min }}$, increases with the number of components. The data are plotted for the unknot $(\emptyset)$, the trefoil $\left(3_{1}\right)$, the square knot $\left(3_{1}^{+} \# 3_{1}^{-}\right)$, the granny knot $\left(3_{1}^{+} \# 3_{1}^{+}\right)$and a compound knot with three trefoil components of the same chirality $\left(3_{1}^{+} \# 3_{1}^{+} \# 3_{1}^{+}\right)$. Oberve the marked difference at small $n$ between the granny and square knots. The granny knot has much more relative entropy than the square knot at low values of $n$.

In Figures 11 and 12 data are given for a collection of prime knots. The data in Figure 12 are for prime knots to six crossings, for $n \in[0,150]$, while these data are also included in Figure 11 for a larger collection of prime knots, for $n \in[0,75]$. The larger scale on the $X$-axis improves the resolution of the data in these graphs.

It is noticable that the ratios approach (within numerical precision) the same limiting curve with increasing $n$ in these graphs. More interesting are the differences between different knot types for small values of $n$ - there are marked and persistent differences between knots with the same minimal crossing numbers (for example $5_{1}$ and $5_{2}$ ), and the ratio $p_{n+2}(K) / p_{n}(K)$ can differ by factors of as large as four or five for different knot types. Most remarkable though are the individual differences 


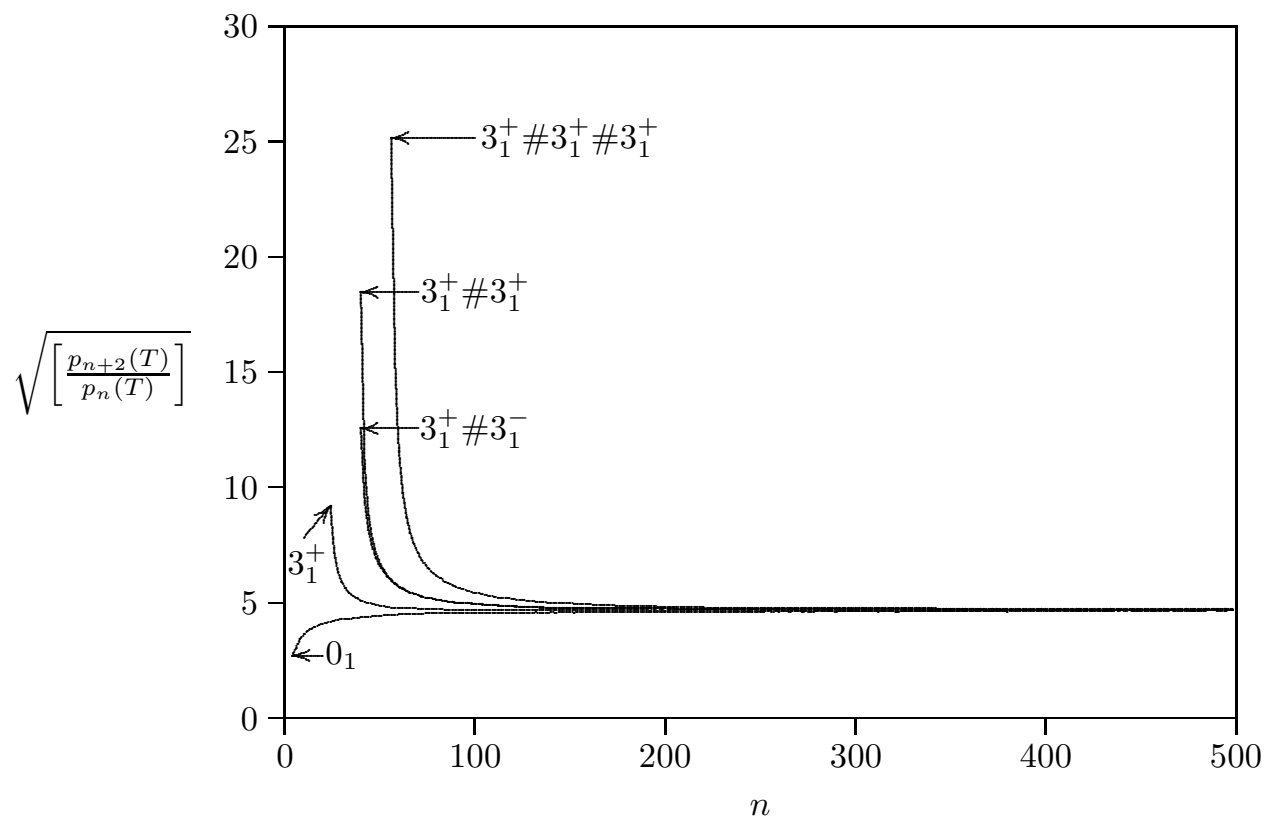

Figure 10. The ratio $\left[p_{n+2}(T) / p_{n}(T)\right]^{1 / 2}$ against $n$ for polygons with knot types the unknot $(\emptyset)$, trefoil $\left(3_{1}\right)$, the square knot $\left(3_{1}^{+} \# 3_{1}^{-}\right)$, the granny knot $\left(3_{1}^{+} \# 3_{1}^{+}\right)$and a compound knot with three trefoil components of the same chirality $\left(3_{1}^{+} \# 3_{1}^{+} \# 3_{1}^{+}\right)$. Oberve the marked difference at small $n$ between the granny and square knots - the granny knot has much more relative entropy than the square knot at low values of $n$.

between different knot types; each knot type appears to have its own characteristic profile in these graphs, and some (such as $7_{4}$ ) presents anomolously large ratios close to its minimal edge number.

\section{Conclusions}

In this paper we presented a numerical method for the calculation of relative entropies of polygons. The method is implemented by defining two statistics (the positive and negative atmospheres) of a polygon. By calculating these statistics, one may determine ratios of the form $p_{n+2} / p_{n}$ in models of polygons, and we have done this in order to estimate the growth constants. For knotted polygons we are able to determine the relative differences in the entropy as a function of the knot types.

This technique is quite general, and can be used on other lattice models, for example lattice walks, trees or animals. In addition, it can also be used to estimate free energies in interacting models of lattice polygons, for example adsorbing polygons or collapsing polygons. We show this in Appendix I below.

In Section 3 we estimated the growth constants of polygons in the square and cubic lattices. We determine $\mu=2.63805 \pm 0.00011$ in the square lattice, and $\mu=4.684092 \pm 0.000042$ in the cubic lattice. These estimates compare well with estimates by other means in equation (8) and equation (10) in the square lattice, and equation (12) in the cubic lattice. While our results are not as accurate in the square lattice, where series analysis of polygon and self-avoiding walk data gave $\mu$ to very 


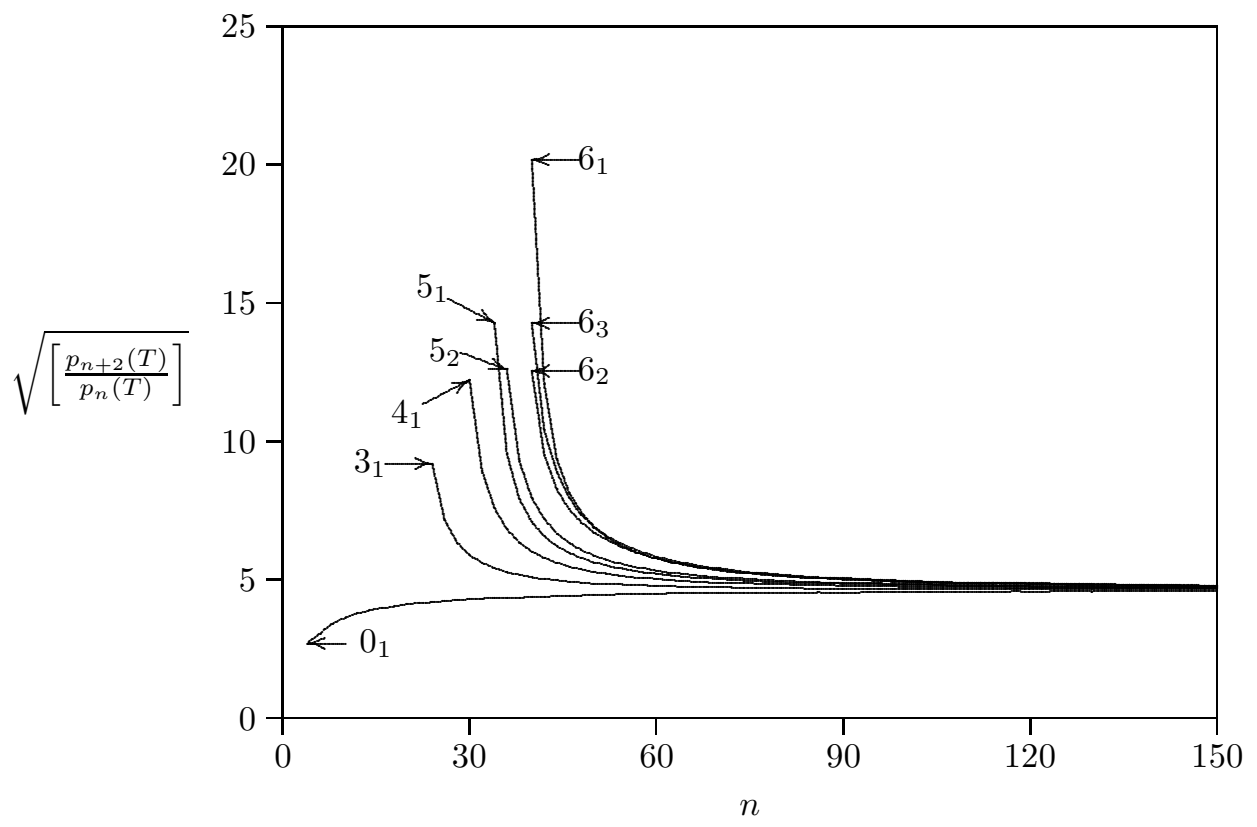

Figure 11. The ratio $\left[p_{n+2}(T) / p_{n}(T)\right]^{1 / 2}$ against $n$ for polygons of six crossing knot types. The most relative entropy is found in $6_{1}$ and the least in the square knot and $6_{2}$, for small values of $n$. Observe that the square knot and $6_{2}$ has virtually the same chagne in relative entropy with increasing $n$.

high accuracy, our result is an independent confirmation of the first digits in those estimates.

In three dimensions our cubic lattice estimate is comparable in accuracy to the recent estimate by Clisby, Liang and Slade [5] in equation (12). We confirm their estimate to four decimal places. We believe that the methods here can be used to find $\mu$ to even higher accuracy in the cubic lattice by using more computer resources.

In Section 4 we considered the relative entropies of polygons of fixed knot types. Little is known about such polygons, but our numerical data show that limits such as in equations (49) and (51) exists. In addition, we plotted those ratios in Figures 9 to 12 , and in each case found that the relative entropy of an unknotted polygon increasing with increasing $n$, while it decreases for a knotted polygon with increasing $n$. In each case it approaches a constant. We also observe that different knots have very different relative entropies at small values of $n$ as in figure 12. This shows that for small values of $n$ these knots will have different statistical properties, a fact which may influence the physical properties of short knotted polymer rings.

\section{Appendix I: Atmospheres of Interacting Polygons}

It is possible to generalise atmospheres to models of polygons weighted by a Boltzmann factor $\beta$. Such models are interacting, and typically have a partition function

$$
Z_{n}(\beta)=\sum_{m} p_{n}(m) \beta^{m}
$$




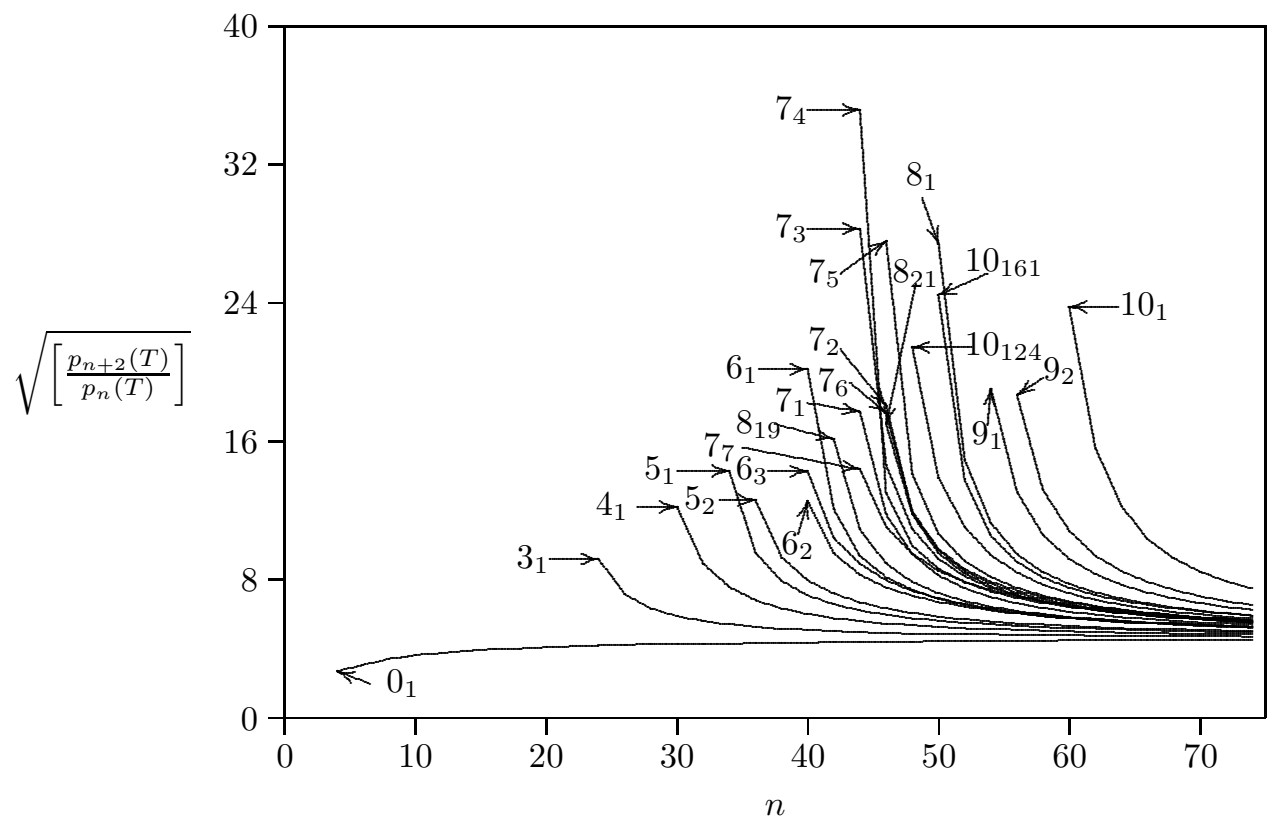

Figure 12. The ratio $\left[p_{n+2}(T) / p_{n}(T)\right]^{1 / 2}$ against $n$ for knotted polygons with crossing number up to ten. crossing knot types. Relative entropy at small values of $n$ is large for $7_{4}, 8_{1}$ and $7_{3}$, amongst these knot types.

where $p_{n}(m)$ is the number of polygons of length $n$ counted up to translational invariance, and with energy $m$ (the number of contacts, visits, span, or the number of occurances of any other property).

In models such as these the construction in Figure 1 changes both the length and the energy of a polygon. Consider a polygon $\omega$ of length $n$ and energy $m(\omega)$, and with $a_{+}(\omega)$ positive atmospheric squares. Similarly, let $\nu$ be a polygon of length $n+2$ and energy $m(\nu)$ and with $\alpha_{-}(\nu)$ negative atmospheric squares.

Suppose that $\nu$ can be obtained from $\omega$ by choosing one atmospheric square in $\omega$, and by applying the construction in Figure 1. Consider now the implementation of the elementary constructions in Figure 1 as the elementary moves in a Metropolis Monte Carlo simulation to sample interacting polygons along a Markov Chain. The transition probability from $\omega$ to $\nu$ in this Markov Chain is

$$
P(\omega \rightarrow \nu)=\frac{1}{\alpha_{+}(\omega)} \min \left\{1, \beta^{m(\nu)-m(\omega)}\right\}
$$

since the probability of choosing a positive atmospheric square is $1 / \alpha_{+}(\omega)$ and since each polygon is weighted by a Boltzmann factor. Reversibility of the Markov Chain requires similarly that

$$
P(\nu \rightarrow \omega)=\frac{1}{\alpha_{-}(\nu)} \min \left\{1, \beta^{m(\omega)-m(\nu)}\right\} .
$$

Assume without loss of generality that $m(\omega) \geq m(\nu)$, then the condition of detailed balance of the algorithm is

$$
P(\omega \rightarrow \nu)=\frac{\alpha_{-}(\nu)}{\alpha_{+}(\omega)} P(\nu \rightarrow \omega) \beta^{m(\nu)-m(\omega)} .
$$


This may be simplified to

$$
\alpha_{+}(\omega) P(\omega \rightarrow \nu) \beta^{m(\omega)}=\alpha_{-}(\nu) P(\nu \rightarrow \omega) \beta^{m(\nu)} .
$$

Sum both sides over $\omega$ (polygons of length $n$ ) and over $\nu$ (polygons of length $n+2$ ). Since $\sum_{\nu} P(\omega \rightarrow \nu)=1$ and $\sum_{\omega} P(\nu \rightarrow \omega)=1$, the result is

$$
\sum_{\omega} \alpha_{+}(\omega) \beta^{m(\omega)}=\sum_{\nu} \alpha_{-}(\nu) \beta^{m(\nu)} .
$$

Dividing this by $Z_{n}(\beta)$ and noting that the weighted average of the atmospheres are given by

$$
\begin{aligned}
& \left\langle\alpha_{+}\right\rangle_{n}=\frac{\sum_{\omega} \alpha_{+}(\omega) \beta^{m(\omega)}}{Z_{n}(\beta)}, \\
& \left\langle\alpha_{-}\right\rangle_{n+2}=\frac{\sum_{\nu} \alpha_{-}(\nu) \beta^{m(\nu)}}{Z_{n+2}(\beta)}
\end{aligned}
$$

then finally produces the result

$$
\frac{Z_{n+2}(\beta)}{Z_{n}(\beta)}=\frac{\left\langle\alpha_{+}\right\rangle_{n}}{\left\langle\alpha_{-}\right\rangle_{n+2}} \approx \frac{\left\langle\alpha_{+}\right\rangle_{n}}{\left\langle\alpha_{-}\right\rangle_{n}}
$$

where one expects the approximation to become more accurate with increasing $n$.

Implementing the above involves the sampling of polygons from a Boltzman distribution

$$
D_{n}(\omega)=\frac{\beta^{m(\omega)}}{Z_{n}(\beta)}
$$

on polygons of length $n$ along a Markov chain, say $\left\{\omega_{i}\right\}_{i=1}^{N}$ (where $m(\omega)$ is the energy of $\omega)$. Then one may estimate $\left\langle\alpha_{+}\right\rangle_{n}$ by forming the average

$$
\left\langle\alpha_{+}\right\rangle_{n} \approx \frac{1}{N} \sum_{i=1}^{N} A_{+}\left(\omega_{i}\right)
$$

where the positive atmosphere of $\omega_{i}$ has $N_{+}\left(\omega_{i}\right)$ unit squares and

$$
A_{+}\left(\omega_{i}\right)=\frac{1}{N_{+}\left(\omega_{i}\right)} \sum_{j=1}^{N_{+}\left(\omega_{i}\right)} \beta^{m\left(\omega_{i j}\right)-m\left(\omega_{i}\right)}
$$

and where $m\left(\omega_{i j}\right)$ is the energy of the polygon if the $j$-th atmospheric square is removed to make a polygon of length $n+2$. A similar definition can be used to define the average negative atmosphere $\left\langle\alpha_{-}\right\rangle_{n+2}$.

Acknowledgments: EJJvR and AR acknowledge support in the form of Discovery Grants from NSERC(Canada).

\section{Bibliography}

[1] Aragão de Carvalho C, Caracciolo S and Fröhlich J 1983 Polymers and $g|\phi|^{4}$-theory in Four Dimensions, Nucl. Phys. B215 [FS7] 209-248

[2] Berg B and Foester D 1981 Random Paths and Random Surfaces on a Digital Computer, Phys. Lett. 106B 323-326

[3] Berretti A and Sokal A D 1985 New Monte Carlo Method for the Self-Avoiding Walk. J. Stat. Phys. 40 483-531 
[4] Broadbent S R and Hammersley J M 1957 Percolation Processes I: Crystals and Mazes. Math. Proc. Camb. Phil. Soc. 53 629-641

[5] Clisby N, Liang R and Slade G 2007 Self-Avoiding Walk Enumeration via the Lace Expansion. J. Phys. A: Math. Theor. 40 10973-11017

[6] de Gennes P G 1979 Scaling Concepts in Polymer Physics. Cornell University Press (New York)

[7] Diao Y 1993 Minimal Knotted Polygons on the Cubic Lattice. J. Knot Theo. Ram. 2413425

[8] Diao Y 1994 The Number of Smallest Knots on the Cubic Lattice. J. Stat. Phys. 74 1247-1254

[9] Flory P J 1955 Statistical Thermodynamics of Semi-Flexible Chain Molecules. Proc. Roy. Soc. (London) A 234 60-73

[10] Flory P J 1969 Statistical Mechanics of Chain Molecules. Wiley Interscience: New York

[11] Grassberger P 1997 Pruned-enriched Rosenbluth Method: Simulation of $\theta$-polymers of Chain Length up to 1000 000. Phys. Rev. E 56 3682-3693

[12] Guida R and Zinn-Justin J 1998 Critical Exponents of the N-vector Model. J. Phys. A: Math. Gen. 31 8103-8121

[13] Guttmann A J and Conway A R 2001 Square Lattice Self-Avoiding Walks and Polygons. Ann. of Comb. 5 319-345

[14] Hammersley J M 1960 Limiting Properties of Numbers of Self-Avoiding Walks. Phys. Rev. 118 656-656

[15] Hammersley J M 1961 The Number of Polygons on a Lattice. Math. Proc. Camb. Phil. Soc. 57 516-523

[16] Hammersley J M and Morton K W 1954 Poor Man's Monte Carlo. J. Roy. Stat. Soc. B 16 23-38

[17] Hara T, Slade G and Sokal A 1993 New Lower Bounds on the Self-Avoiding-Walk Connective Constant. J. Stat. Phys. 72 3/4 479-517

[18] Janse van Rensburg E J 1992 Ergodicity of the BFACF Algorithm in Three Dimensions, J. Phys. A: Math. Gen. 25 1031-1042

[19] Janse van Rensburg E J and Promislow S D 1995 Minimal Knots in the Cubic Lattice. J. Knot Theo. Ram. 4 115-130

[20] Janse van Rensburg E J and Rechnitzer A 2003 High Precision Canonical Monte Carlo Determination of the Connective Constant of Self-Avoiding Trees. Phys. Rev. E 67036116 $1-9$

[21] Janse van Rensburg E J and Whittington S G 1991 The BFACF Algorithm and Knotted Polygons. J Phys. A: Math. Gen. 24 5553-5567

[22] Janse van Rensburg E J, Whittington S G and Madras N 1990 The Pivot Algorithm and Polygons: Results on the FCC Lattice. J Phys. A: Math. Gen. 23 1589-1612

[23] Jensen I and Guttmann A J 1998 Self-Avoiding Walks, Neighbour-Avoiding Walks and Trials on Semi-Regular Lattices. J. Phys. A: Math. Gen. 31 8137-8145

[24] Jensen I and Guttmann A J 1999 Self-Avoiding Polygons on the Square Lattice. J. Phys. A: Math. Gen. 32 4867-4876

[25] Kesten H 1963 On the Number of Self-Avoiding Walks. J. Math. Phys. 4 960-969

[26] Kesten H 1964 On the Number of Self-Avoiding Walks II. J. Math. Phys. 5 1128-1137

[27] Le Guillou J C and Zinn-Justin J 1989 Accurate Critical Exponents from Field Theory. J. de Physique 50 1365-1370

[28] Madras N, Orlitsky A and Shepp L A 1990 Monte Carlo Generation of Self-Avoiding Walks with Fixed Endpoints and Fixed Length. J. Stat. Phys. 58 159-183

[29] Madras N and Sokal A D 1988 The Pivot Algorithm: A Highly Efficient Monte Carlo Method for the Self-Avoiding Walk. J. Stat. Phys. 47 573-595

[30] Madras N and Slade G 1993 The Self-Avoiding Walk. (Birhäuser: Boston)

[31] Michels J P J and Wiegel F W 1984 Probability of Knots in a Polymer Ring. Phys. Lett. 90A 381-384

[32] Michels J P J and Wiegel F W 1986 On the Topology of a Polymer Ring. Proc. Roy. Soc. (London) A 403 269-284

[33] Nienhuis B 1982 Exact Critical Point and Critical Exponents on $O(n)$ Models in Two Dimensions. Phys. Rev. Lett. 49 1062-1065

[34] Nienhuis B 1984 Coulomb Gas Formulation of the Two-Dimensional Phase Transitions. In Phase Transitions and Critical Phenomena 11 1-53. Eds. C. Domb and J.L. Lebowitz (Academic Press)

[35] Nidras P 1996 Grand Canonical Simulations of the Interacting Self-Avoiding Walk Model. J. Phys. A: Math. Gen. 29 7929-7942

[36] O'Brien G L 1990 Monotonicity of the Number of Self-Avoiding Walks. J. Stat. Phys. 59 969-979

[37] Orlandini E, Tesi M C, Janse van Rensburg E J and Whittington S G 1998 Asymptotics of Knotted Lattice Polygons, J. Phys. A: Math. Gen. 31 5953-5967. 
[38] Pippenger N 1989 Knots in Self-Avoiding Walks. Disc. Appl. Math. 25 273-278

[39] Prellberg T and Owczarek A L 2000 Four-dimensional Polymer Collapse: Pseudo-first-order Transition in Interacting Self-Avoiding Walks. Phys. Rev. E 62 3780-3789

[40] Rechnitzer A and Janse van Rensburg 2002 Canonical Monte Carlo Determination of the Connective Constant of Self-Avoiding Walks. J. Phys. A: Math. Gen. 35 L605-L612

[41] Sumners D W and Whittington S G 1988 Knots in Self-Avoiding Walks. J. Phys. A: Math. Gen. 21 1689-1694 